\title{
Impacto cardiovascular em pacientes infectados com o SARS-COV-2 (COVID-19)
}

\author{
Cardiovascular impact in patients infected with SARS-COV-2 (COVID-19)
}

Impacto cardiovascular en pacientes infectados por SARS-COV-2 (COVID-19)

Felipe de Oliveira Benigno ${ }^{1 *}$, Alice Neres Bezerra do Nascimento ${ }^{1}$, Cinthia Millena Cabral Costa ${ }^{1}$, Wadja Oliveira Leite dos Ramos ${ }^{1}$, Hirla Vanessa Soares de Araújo.

\section{RESUMO}

Objetivo: Identificar na literatura científica o que as evidências apontam sobre as implicações cardiovasculares em pacientes acometidos pelo COVID-19. Métodos: Trata-se de uma revisão integrativa da literatura, com a finalidade de reunir e sintetizar resultados de investigações sobre o tema. Para a seleção dos estudos, foram pesquisadas as bases de dados Scientific Eletronic Library Online (SciELO) e Medical Literature Analysis and Retrievel System Online (Medline) no período de março a maio de 2021. Os descritores utilizados foram: Coronavirus Infections AND Complications Cardiovascular. A amostra final consistiu em 15 artigos analisados. Resultados: As infecções virais aumentam a chance de o paciente desenvolver complicações cardiovasculares, além disso, os pacientes com algum tipo de doença cardiovascular estão mais vulneráveis à influenza e outras doenças infecciosas que afetam o trato respiratório. Grande parte dos estudos relacionados ao SARS-COV- 2 estão relacionados ao seu impacto pulmonar, no entanto, as doenças cardiovasculares também estão associadas a pior prognóstico e a uma progressão mais grave da COVID-19. Considerações finais: São necessários estudos clínicos randomizados para esclarecer os mecanismos fisiopatológicos da covid-19 no organismo humano e as medidas assistenciais e terapêuticas para serem adotadas a fim de melhorar o prognóstico da doença, reduzindo a mortalidade.

Palavras-chave: Sars-cov-2, Infecção coronavírus, Covid-19, Doenças cardiovasculares.

\begin{abstract}
Objective: To identify in the scientific literature what evidence points to the cardiovascular implications in patients affected by COVID-19. Methods: This is an integrative literature review, with the purpose of gathering and synthesizing research results on the subject. To select the studies, the Scientific Electronic Library Online (SciELO) and Medical Literature Analysis and Retrievel System Online and (Medline) databases were searched from march to may 2021. The descriptors used were: Coronavirus Infections AND Complications Cardiovascular. The final sample consisted of 15 articles analyzed. Results: Viral infections increase the chance of the patient to develop cardiovascular complications, in addition, patients with some type of cardiovascular disease are more vulnerable to influenza and other infectious diseases that affect the respiratory tract. Most studies related to SARS-COV-2 are related to its pulmonary impact, however, cardiovascular diseases are also associated with a worse prognosis and a more severe progression of COVID-19. Final considerations: Randomized clinical trials are needed to clarify the pathophysiological mechanisms of covid-19 in the human body and the care and therapeutic measures to be adopted in order to improve the prognosis of the ase, reducing mortality.
\end{abstract}

Key words: Sars-cov-2, Coronavirus infection, Covid-19, Cardiovascular diseases.

${ }^{1}$ Centro Universitário dos Guararapes (UNIFG), Recife - PE. *E-mail: felipe92oliveira@gmail.com

SUBMETIDO EM: 6/2021

ACEITO EM: 7/2021

PUBLICADO EM: 8/2021 


\section{RESUMEN}

Objetivo: Identificar en la literatura científica qué evidencia apunta a las implicaciones cardiovasculares en pacientes afectados por COVID-19. Métodos: Se trata de una revisión integradora de la literatura, con el objetivo de recopilar y sintetizar los resultados de la investigación sobre el tema. Para seleccionar los estudios, se realizaron búsquedas en las bases de datos Scientific Electronic Library Online (SciELO) y Medical Literature Analysis and Retrievel System Online y (Medline) de marzo a mayo de 2021. Los descriptores utilizados fueron: Infecciones por coronavirus $Y$ complicaciones cardiovasculares. La muestra final estuvo formada por 15 artículos analizados. Resultados: Las infecciones virales aumentan la posibilidad del paciente de desarrollar complicaciones cardiovasculares, además, los pacientes con algún tipo de enfermedad cardiovascular son más vulnerables a la influenza y otras enfermedades infecciosas que afectan el tracto respiratorio. La mayoría de los estudios relacionados con el SARS-COV-2 están relacionados con su impacto pulmonar, sin embargo, las enfermedades cardiovasculares también se asocian con un peor pronóstico y una progresión más severa de COVID-19. Consideraciones finales: Se necesitan ensayos clínicos aleatorizados para aclarar los mecanismos fisiopatológicos de covid-19 en el cuerpo humano y las medidas asistenciales y terapéuticas a adoptar para mejorar el pronóstico de la enfermedad, reduciendo la mortalidad.

Palabras clave: Sars-cov-2, Infección por coronavirus, Covid-19, Enfermedad cardiovascular.

\section{INTRODUÇÃO}

Segundo a Organização Mundial da Saúde (OMS), os vírus do tipo Corona são de uma grande família que causam doenças que afetam principalmente o sistema respiratório, podendo ir desde resfriados comuns até sintomas mais severos como a Síndrome Respiratória Aguda Grave (SARS-CoV-1) e a Síndrome Respiratória do Oriente Médio (MERS-CoV). Tais epidemias ocorreram em 2002 na China e 2012 na Península Arábica, respectivamente (OMS, 2021).

O primeiro caso do novo Coronavírus (COVID-19) foi reportado e identificado na cidade de WuhanChina, em meados de dezembro de 2019, onde se espalhou rapidamente pelo planeta, em 11 de março de 2020 foi decretada pandemia pela OMS, atualmente, o Brasil acumula mais de 12 milhões de casos confirmados e 300 mil óbitos por COVID-19 (OMS, 2021).

Grande parte das pessoas que são infectadas pelo vírus apresentam sintomas de leve a grave, como febre, tosse seca, fadiga e dificuldade de respirar, outros sintomas como diarreia, lombalgia, dor precordial, anosmia já foram descritos em pacientes que foram infectados pelo COVID-19. Os sintomas podem aparecer de 2 a 14 dias depois da infecção e o indivíduo poderá se recuperar sem necessitar de tratamento especial. Embora grande parte do foco esteja nas complicações pulmonares (onde as complicações respiratórias estão fortemente associadas com a mortalidade), o SARS-CoV-2 pode causar também lesões no fígado, rins, coração e veias, sendo as complicações cardiovasculares um grande contribuidor para a taxa de mortalidade associada à COVID-19 (BRASIL, 2020).

As comorbidades como doenças cardiovasculares, doenças pulmonares, renais, diabetes, hipertensão, idade avançada, imobilidade, choque cardiogênico e a coagulopatia são alguns dos fatores de risco associados ao COVID-19. Entre os citados, o choque cardiogênico pode ocorrer naqueles pacientes em situação crítica além de ser a complicação mais grave da infecção (LONG B, et al., 2020).

O paciente infectado tende a desenvolver após 15 dias do início dos sintomas alguma complicação cardiovascular, dentre elas as mais relatadas estão à miocardite fulminante ou uma disfunção miocárdica que contribuem para o aparecimento de arritmias. A hipertensão parece estar associada a um curso mais grave da doença e o paciente com esse tipo de comorbidade é mais propenso a evoluir para a síndrome respiratória aguda grave. A lesão miocárdica ocorre em $17 \%$ dos pacientes infectados, na forma de miocardite aguda ou como uma lesão secundária devido à baixa demanda de oxigênio levando ao infarto agudo do miocárdio (GUZIK TJ, et al., 2020). 
No contexto do COVID-19, algumas intervenções realizadas no início do atendimento são de grande importância na abordagem do paciente, intervenções como a realização de eletrocardiograma e ecocardiograma são de suma importância, pois as anormalidades nesses exames são sinais de gravidade da doença e estão associadas a mau prognóstico (SHI S, et al., 2020).

Por ser uma doença nova, ainda não possui um tratamento especifico e mesmo com o inicio da vacinação ainda é necessário que as medidas de prevenção da doença ainda sejam mantidas, principalmente em países onde a vacinação em massa ainda não foi concluída. Para isso, a OMS recomenda a o uso de máscaras e realização do distanciamento social, além de medidas de higienização das mãos com água e sabão, ou álcool a 70\%, a fim de retardar a propagação da doença (BRASIL, 2020).

É justificada a preocupação e a atenção, especialmente dos profissionais de diversas áreas da equipe multidisciplinar para subsidiar as boas práticas e o manejo adequado frente a esses pacientes. Logo, o objetivo deste trabalho é identificar na literatura científica o que as evidências apontam sobre as implicações cardiovasculares em pacientes acometidos pelo COVID-19.

\section{MÉTODOS}

Trata-se de uma revisão integrativa da literatura, com a finalidade de reunir e sintetizar resultados de investigações sobre o tema, conduzida no intuito de atender à questão norteadora: "O que as evidências apontam sobre os eventos cardiovasculares em pacientes diagnosticados com COVID-19?". A partir desse questionamento, procederam-se as demais etapas para realização da revisão: onde foi realizada os critérios de inclusão, definição das informações das pesquisas selecionadas, avaliação dos estudos incluídos na revisão integrativa, interpretação dos resultados e síntese dos resultados obtidos (BRIZOLA J, 2016).

Para a seleção dos estudos, foram pesquisadas as bases de dados Scientific Eletronic Library Online (SciELO), National Center for Biotechnology Information (PubMed) e Medical Literature Analysis and Retrievel System Online (Medline) no período de março a maio de 2021. As bases de dados foram escolhidas pela sua grande abrangência de estudos. Realizou-se a seleção dos descritores após consulta ao DeCS e MeSH. Os descritores/palavras chaves utilizados foram: Coronavirus Infections AND Complications Cardiovascular

Obedeceu-se a mesma sequência na inserção dos descritores nas bases de dados para as buscas. Os artigos selecionados atenderam aos seguintes critérios de inclusão: pesquisas disponíveis eletronicamente nas bases de dados selecionadas, publicadas em periódicos revisados por pares, sobre os eventos cardiovasculares em pacientes diagnosticados com COVID-19, textos completos disponíveis gratuitamente nos idiomas português, inglês e espanhol e publicados nos últimos 5 anos (2017-2021). A busca nas bases de dados e no portal com a combinação dos descritores citados resultou em 138 artigos, sendo 22 no SciELO, 206 na PubMed e 116 na Medline.

A leitura seletiva dos artigos foi realizada, inicialmente, com análise do título e resumo. Os artigos duplicados foram registrados apenas uma vez. Para garantir o registro conjunto de informações relevantes ao tema, foi utilizado instrumento próprio para análise dos artigos, o qual incluía dados de identificação (período publicado, formação e instituição à qual os autores estão vinculados, idioma e país), aspectos metodológicos dos estudos (delineamento da pesquisa, amostragem e tratamento dos dados) e principais resultados e conclusões.

Em seguida, realizou-se análise detalhada dos artigos, considerando o rigor e as características dos mesmos, seguindo-se o registro das informações elencadas no instrumento. As informações foram interpretadas, resumidas e organizadas em quadro síntese, comparando-se os resultados, e finalizadas com proposições acerca dos estudos, essas informações encontra-se na Figura 1.

Inicialmente foi encontrado um total de 344 artigos científicos. Após a leitura dos títulos e resumos, foram excluídas 298 publicações em duplicata e por não responderem à pergunta norteadora. Em seguida, foi realizada a leitura de 46 publicações na íntegra. Destas, 31 foram excluídas por não responderem à 
questão norteadora da revisão integrativa. Assim, foi obtido um total de 15 artigos científicos voltados aos eventos cardiovasculares em pacientes diagnosticados com COVID-19. Os artigos selecionados foram submetidos a uma classificação quanto ao seu nível de evidência, foram priorizados estudos que apresentassem revisão sistemática ou estudo de meta-analise, opinião de autoridades ou relatório de comitês de especialistas, revisão sistemática de estudos descritivos e qualitativos, estudos de coorte e caso controle e ensaio clínico randomizado, controlado, bem delineado (STILLWELL SB, et al., 2010).

Figura 1 - Fluxograma da seleção dos artigos para esta revisão integrativa.

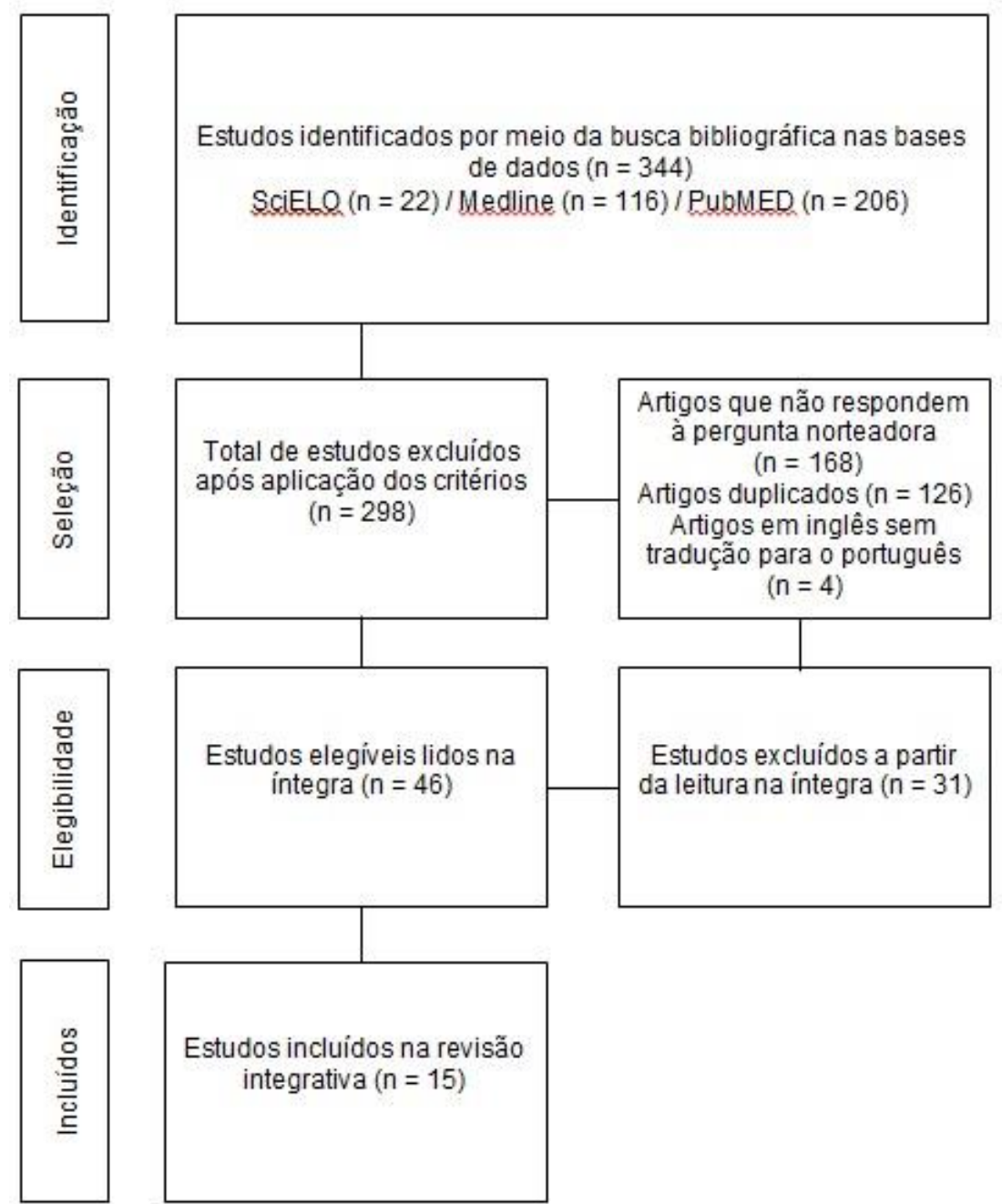

Fonte: Benigno FO, et al., 2021.

\section{RESULTADOS}

Dos 15 artigos que compuseram a amostra todos estavam disponíveis nos idiomas inglês e português, publicados entre os anos de 2019 a 2021. Em relação ao local onde ocorreram as pesquisas, os estudos foram desenvolvidos nos Estados Unidos da América, China e Inglaterra. O quadro 1 destaca o ano de publicação do estudo, o método utilizado para fundamentar as pesquisas e os principais resultados.

A partir da análise dos objetivos dos artigos incluídos observou-se que: um artigo teve o objetivo de descrever as características clínicas e epidemiológicas relacionadas ao COVID-19; oito artigos tiveram como objetivo descrever as implicações cardíacas do SARS-COV-2 e compreender os danos causados por ele ao sistema cardiovascular; três artigos tiveram como objetivo principal descrever a fisiopatologia da doença; e outros três artigos descrever as intervenções necessárias frente a pandemia do COVID-19, e o manejo ao paciente crítico. 
Quadro 1 - Síntese dos principais achados.

\begin{tabular}{|c|c|c|}
\hline $\mathbf{N}$ & Autores (Ano) & Principais achados \\
\hline 1 & SAVALAN BF, et al., 2020 & $\begin{array}{l}\text { Descritivo. A infecção por COVID-19 tem sido associada a lesão } \\
\text { miocárdica. }\end{array}$ \\
\hline 2 & LONG B, et al., 2020 & $\begin{array}{l}\text { Descritivo. Lesão miocárdica e miocardite com elevação da troponina } \\
\text { está relacionado ao aumento do estresse fisiológico cardíaco, } \\
\text { hipóxia ou lesão miocárdica direta. } \\
\text { Alguns medicamentos utilizados para tratar a infecção pelo COVID- } \\
19 \text { apresentam efeitos cardiovasculares que podem levar a } \\
\text { complicações cardíacas potenciais. }\end{array}$ \\
\hline 3 & GUZIK TJ, et al., 2020. & $\begin{array}{l}\text { Descritivo. A avaliação precoce e o monitoramento contínuo de dano } \\
\text { cardíaco após a hospitalização podem identificar pacientes com } \\
\text { lesão cardíaca e prever complicações COVID-19. } \\
\text { Considerações cardiovasculares das terapias atualmente usadas, } \\
\text { bem como terapias experimentais }\end{array}$ \\
\hline 4 & FACONTI L, et al., 2020. & $\begin{array}{l}\text { Descritivo. Pacientes com doença cardiovascular (DCV), incluindo } \\
\text { IC, são particularmente vulneráveis à influenza e outras doenças } \\
\text { infecciosas do trato respiratório superior e inferior e apresentam } \\
\text { maior risco de desfechos desfavoráveis. }\end{array}$ \\
\hline 5 & CHENG P, et al., 2020. & $\begin{array}{l}\text { Descritivo. Evidências epidemiológicas emergentes sugerem que os } \\
\text { fatores de risco cardiovascular estão associados ao aumento da } \\
\text { gravidade da doença e da mortalidade em pacientes com COVID- } 19 \text {. } \\
\text { Pacientes com uma forma mais grave de COVID-19 também têm } \\
\text { maior probabilidade de desenvolver complicações cardíacas, como } \\
\text { lesão miocárdica e arritmia. }\end{array}$ \\
\hline 6 & AGHAGOLI G, et al., 2020. & $\begin{array}{l}\text { Descritivo. As doenças cardiovasculares também estão associadas a } \\
\text { pior prognóstico e progressão mais graves da COVID-19 }\end{array}$ \\
\hline 7 & ZHENG YY, et al., 2020. & $\begin{array}{l}\text { Descritivo. Paciente com sintomas graves tendem a ter complicações } \\
\text { envolvendo lesão miocárdica aguda. }\end{array}$ \\
\hline 8 & WANG D, et al., 2020. & $\begin{array}{l}\text { Editorial. } 17 \% \text { dos pacientes desenvolveram algum tipo de arritmia } \\
\text { como fibrilação atrial, bloqueio de condução, e taquicardia } \\
\text { ventricular. }\end{array}$ \\
\hline 9 & ZHOU F, et al., 2020. & $\begin{array}{l}\text { Descritivo. Mecanismo de infecção e a patogenicidade do SARS- } \\
\text { CoV-2 }\end{array}$ \\
\hline 10 & CHEN Y, et al., 2020. & $\begin{array}{l}\text { Descritivo. O SARS-CoV-2 pode fazer a desregulação do ECA2, } \\
\text { causando um acúmulo excessivo tóxico da angiotensina-2 no plasma }\end{array}$ \\
\hline 11 & $\begin{array}{l}\text { MARTINS JDN, et al., } \\
2020 \text {. }\end{array}$ & $\begin{array}{l}\text { Descritivo. Fatores patológicos do vírus e da pré-existência de } \\
\text { comorbidades ligadas ao sistema cardiovascular como Doença } \\
\text { Cardiovascular (DCV) e hipertensão arterial, apontam desfechos } \\
\text { desfavoráveis, }\end{array}$ \\
\hline 12 & TANG N, et al., 2020. & $\begin{array}{l}\text { Descritivo. Terapia de anticoagulação, especialmente com heparina } \\
\text { de baixo peso molecular, pode estar relacionada à diminuição da } \\
\text { mortalidade por infecção grave pela covid- } 19 \text {. }\end{array}$ \\
\hline 13 & XIE Y, et al., 2020. & $\begin{array}{l}\text { Descritivo. O Tromboembolismo venoso (TEV) está presente na } \\
\text { maioria dos pacientes infectados que desenvolvem a forma grave da } \\
\text { doença }\end{array}$ \\
\hline 14 & CHAVEZ S, et al., 2020. & $\begin{array}{l}\text { Descritivo. Interações farmacológicas de alguns fármacos utilizados } \\
\text { no tratamento da COVID-19. }\end{array}$ \\
\hline 15 & SHI S, et al., 2020. & $\begin{array}{l}\text { Descritivo. Intervenções realizadas como a realização de } \\
\text { eletrocardiograma e ecocardiograma são de suma importância, pois } \\
\text { as anormalidades nesses exames são sinais de gravidade da } \\
\text { doença e estão associadas a mau prognóstico. }\end{array}$ \\
\hline
\end{tabular}

Fonte: Benigno FO, et al., 2021. 


\section{DISCUSSÃO}

O agente responsável pela COVID-19, o SARS-CoV-2, é um vírus pertencente à família Coronaviridae, o qual apresenta elevada similaridade, tanto ao se tratar de estrutura como em sintomas clínicos, com o vírus causador do surto de SARS em 2003, o SARS-CoV. Estudos revelam que existe cerca de $80 \%$ de homologia entre o SARS-CoV e o SARS-CoV-2 (CERAOLO C e GIORGI FM, 2020). Essa similaridade entre os dois tornou possível que cientistas especulassem que o mecanismo de infecção e a patogenicidade do SARS-CoV-2 seriam parecidos. Através de um estudo foi comprovado que assim como o SARS-CoV, o novo coronavírus utiliza o mesmo receptor celular proteico, a Enzima Conversora de Angiotensina 2 (ECA2), como porta de entrada do vírus na célula (ZHOU F, et al., 2020).

O ECA2 tem níveis de expressão altos em outros órgãos além dos pulmões, como o coração, o esôfago, os rins, a bexiga e o íleo, o que justifica a maior vulnerabilidade à infecções extrapulmonares da doença como falência renal e miocardites (ZHOU F, et al., 2020). Evidências de outros coronavírus sugerem que o SARS-CoV-2 pode fazer a desregulação do ECA2, causando um acúmulo excessivo tóxico da angiotensina2 no plasma, o que induz a dois desfechos principais e graves: a síndrome do desconforto respiratório agudo e miocardite fulminante (CHEN Y, et al., 2020; HANFF TC, et al., 2020).

Por estar presente em níveis altos de expressão no coração e ser o receptor funcional para o vírus, 0 ECA2 causa desequilíbrio do sistema renina angiotensina, uma vez que a angiotensina 1-7 (Ang 1-7), convertida pela ECA2 a partir de angiotensina 2 (Ang II) resulta em efeitos protetores cardiovasculares. Assim, quando ocorre a diminuição da atividade de ECA2 no coração devido à ação do vírus, ocorre aumento da Ang II, que não é mais convertida em Ang 1-7 e deixam de ter os efeitos anti-fibrose, vasodilatador, anti-insuficiência cardíaca, antitrombose, anti-hipertrofia miocárdica, antiarrítmica; resultando em vasoconstrição, efeitos pró-inflamatórios e pró-fibróticos, levando a desfechos cardiovasculares negativos (MARTINS JDN, et al., 2020).

Além dos fatores patológicos do vírus e da pré-existência de comorbidades ligadas ao sistema cardiovascular como Doença Cardiovascular (DCV) e hipertensão arterial, serem influenciadores de desfechos desfavoráveis, a hipóxia também é um fator de lesão do sistema cardiovascular. Uma vez que o vírus da COVID-19 causa lesão pulmonar que leva à hipoxemia, situação essa onde a Pressão Parcial de Oxigênio ( $\mathrm{PaO} 2$ ) e a Saturação de Oxigênio (SatO2) decaem na corrente sanguínea, levando consequentemente à acumulação de ácido lático e metabólitos os quais causam lesões celulares no miocárdio (MARTINS JDN, et al., 2020).

As infecções virais podem causar efeitos deletérios ao sistema cardiovascular, segundo o Centro de Controle e Prevenção de Doenças $(C D C)$ as infecções virais são uma das causas mais frequentes de miocardite infecciosa, um estudo relatou que as infecções virais aumentaram a chance de o paciente desenvolver arritmias, Insuficiência Cardíaca e a Síndrome coronariana aguda (SCA), além disso, os pacientes com algum tipo de doença cardiovascular estão mais vulneráveis à influenza e outras doenças infecciosas que afetam o trato respiratório, isso pode acontecer por conta da invasão direta do vírus ao sistema cardiovascular, ou pelas respostas sistêmicas como uma reação imunológica ou redução da demanda de oxigênio ao organismo (FACONTI L, et al., 2020).

Grande parte dos estudos relacionados ao SARS-COV- 2 estão relacionados ao seu impacto pulmonar, no entanto, em uma revisão foi apontado que as doenças cardiovasculares também estão associadas a pior prognóstico e a uma progressão mais grave da COVID-19, isso por conta que os pacientes maiores de sessenta anos ou que possuem alguma comorbidade como doença coronariana, hipertensão ou diabetes, possuem um risco aumentado de contrair a covid-19 (AGHAGOLI G, et al., 2020).

Um estudo apontou em seus resultados que os pacientes infectados com o COVID-19, possuem uma alta incidência de sintomas cardiovasculares, devido a resposta inflamatória sistêmica da doença ao sistema corporal e a distúrbios do sistema imunológico que também tendem aumentar durante a progressão da doença. Sendo assim os pacientes que possuem algum tipo de doenças cardiovasculares podem apresentar um pior prognostico da doença quando são infectados e mesmo durante o tratamento da COVID-19 é necessária uma atenção com relação a proteção do sistema cardiovascular (FERRARI F, 2020). 
O estudo que trata das evidências epidemiológicas do COVID-19 mostrou que as evidências sugerem que os fatores de riscos cardíacos estão associados ao aumento da gravidade da doença e da mortalidade nos pacientes. Os pacientes que desenvolvem a forma grave da doença necessitando de cuidados intensivos possuem uma probabilidade maior de desenvolver complicações cardíacas, como lesão miocárdica e arritmia (CHENG P, et al., 2020). Um relatório publicado na China identificou em uma amostra de 138 pacientes de Wuhan, que 17\% desses pacientes desenvolveram algum tipo de arritmia como fibrilação atrial, bloqueio de condução, e taquicardia ventricular (WANG D, et al., 2020). A incidência dos sintomas cardiovasculares em pacientes diagnosticados com covid-19 é alta, devido à resposta inflamatória sistêmica e distúrbios do sistema imunológico durante a progressão da doença (ZHENG YY, et al., 2020).

Um estudo que aborda que a infecção por COVID-19 tem sido associada à lesão miocárdica apontou que a lesão ao miocárdio foi observada nos pacientes infectados pelo vírus, alguns casos com infecções anteriores com o SARS já foram associados a complicações cardíacas como disfunção sistólica e diastólica com subsequente insuficiência cardíaca, arritmias e morte súbita devido à lesão miocárdica (SAVALAN BF, et al., 2020).

Em um estudo que avaliou o monitoramento contínuo do dano cardíaco, relatou que devido à fisiopatologia da COVID-19 o paciente infectado tende a desenvolver após 15 dias do início dos sintomas alguma complicação cardiovascular, dentre elas as mais relatadas estão à miocardite fulminante ou uma disfunção miocárdica que contribuem para o aparecimento de arritmias. A hipertensão parece estar associada a um curso mais grave da doença e o paciente com esse tipo de comorbidade é mais propenso a evoluir para a síndrome respiratória aguda grave. O estudo relata também que a lesão miocárdica ocorre em $17 \%$ dos pacientes infectados, na forma de miocardite aguda ou como uma lesão secundária devido à baixa demanda de oxigênio levando ao infarto agudo do miocárdio (GUZIK TJ, et al., 2020).

No mesmo estudo que avaliou as lesões miocárdicas, observou-se que os casos dos pacientes com arritmias apresentaram também elevação na troponina e mioglobina e consequentemente aumento no índice de mortalidade, com relação a esses biomarcadores, foi analisado também que pacientes com histórico de doença cardiovascular eram mais suscetíveis a ter os níveis desses marcadores elevados e a ter lesão cardíaca (SAVALAN BF, et al., 2020).Os Níveis de troponina I que excedem o limite de referência são observados em $8 \%$ a $12 \%$ dos casos de COVID-19 positivos (FACONTI L, et al., 2020).

Um estudo comparou os níveis de troponina nos pacientes infectados e descreveu que em uma amostra de 41 pacientes 05 apresentaram lesão miocárdica com aumento de alta sensibilidade nos níveis de troponina (HUANG C, et al. 2020). A medição precoce de biomarcador de dano cardíaco no paciente admitido ajuda a identificar possível dano miocárdico (FACONTI L, et al., 2020). A Lesão miocárdica e a miocardite com elevação da troponina observadas nos pacientes infectados estão relacionadas ao aumento do estresse fisiológico cardíaco, hipóxia ou lesão miocárdica direta (LONG B, et al., 2020).

Pacientes com insuficiência cardíaca apresentam risco aumentado de eventos agudos ou exacerbação da doença de base, quando infectados. E a coagulação intravascular disseminada e embolia pulmonar foram altamente prevalentes na COVID-19 (GUZIK TJ, et al., 2020). O Tromboembolismo venoso (TEV) está presente na maioria dos pacientes infectados que desenvolvem a forma grave da doença (XIE Y, et al., 2020; DANZI GB, 2020). Entre os fatores potenciais estão a inflamação sistêmica, estado de coagulação anormal, disfunção de múltiplos órgãos e doenças graves, todos esses aumentam o risco de TEV (ZHOU F, et al., 2020; WANG D, et al., 2020; CHEN J, 2020). Um estudo mostrou que a terapia de anticoagulação, especialmente com heparina de baixo peso molecular, pode estar relacionada à diminuição da mortalidade por infecção grave pela covid-19 (TANG N, et al., 2020).

No contexto do COVID-19, intervenções realizadas como a realização de eletrocardiograma e ecocardiograma são de suma importância, pois as anormalidades nesses exames são sinais de gravidade da doença e estão associadas a mau prognóstico (SHI S, et al., 2020; GUO T, et al., 2020).

Muitos dos fármacos estudados para o tratamento medicamentoso da COVID-19 têm ampla interação com outros medicamentos cardiovasculares, incluindo anti-hipertensivos, medicamentos antiarrítmicos, anticoagulantes, medicamentos antiplaquetários e estatinas (DRIGGIN E, et al., 2020). Os medicamentos 
atuais sob investigação incluem antivirais (remdesivir; ribavirina; lopinavir/ritonavir; favipiravir), os antimaláricos (cloroquina; hidroxicloroquina), antibióticos (azitromicina), corticosteróides e produtos biológicos como tocilizumabe (CHAVEZ S, et al., 2020; ELFIKY AA, 2020).

Alguns fármacos que são utilizados no tratamento apresentam efeitos adversos e algumas interações, como no caso do Lopinavir/ritonavir que pode causar prolongamento do intervalo QT e PR na visualização do Eletrocardiograma (ECG). Esses fármacos podem também afetar os medicamentos anticoagulantes, agentes plaquetários e estatinas. A cloroquina e hidroxicloroquina prejudicam o $\mathrm{Ph}$ intracelular resultando assim em distúrbios eletrolíticas, cardiotoxicidades e intervalos de QT prolongados, atuando também com agentes antiarrítmicos (PAGE RL, et al., 2016; TONNESMANN E, et al., 2013). A metilprednisolona é capaz de ocasionar anormalidades eletrolíticas, retenção de líquidos e hipertensão. O antibiótico azitromicina pode ocasionar interações com anticoagulantes, antiarrítmicos, estatinas, além de ocasionar um prolongamento no intervalo QT, com relação ao seu efeito adverso pode levar a uma arritmia (LIU K, et al., 2020).

Ainda é difícil apresentar os danos cardiovasculares crônicos em pacientes que foram diagnosticados com a infecção, um estudo que analisou as doenças cardiovasculares que os pacientes infectados desenvolveram apontou que baseado no SARS-COV semelhante biologicamente ao SARS-COV-2, relatou que pacientes infectados desenvolveram doenças cardiovasculares e alterações metabólicas séricas, no entanto fazem-se necessárias outras investigações do impacto cardiovascular pós COVID-19. A avaliação regular do risco cardiovascular deve ocorrer em todos os pacientes diagnosticados com covid-19, nos pacientes que sobreviveram à infecção é necessária a avaliação periódica devido às consequências em longo prazo que podem ocorrer (AGHAGOLI G, et al., 2020).

\section{CONSIDERAÇÕES FINAIS}

Grande parte dos estudos estão relacionados ao impacto pulmonar que o vírus causa ao organismo, no entanto é importante observar as complicações cardiovasculares que auxiliam ao aumento da mortalidade, tendo em vista que essas complicações estão presente em grande parte dos pacientes infectados com o COVID-19, devido a sua fisiopatologia, a qual ainda precisa de mais estudos para esclarecimento, e também frente ao tratamento utilizado o qual pode desenvolver efeitos adversos ao paciente. São necessários estudos clínicos randomizados para esclarecer os mecanismos fisiopatológicos da COVID-19 no organismo humano e as medidas assistenciais e terapêuticas para serem adotadas a fim de melhorar o prognóstico da doença, reduzindo a mortalidade.

\section{REFERÊNCIAS}

1. AGHAGOLI G, et al. Cardiac involvement in COVID-19 patients: Risk factors, predictors, and complications: A review. Journal of cardiac surgery. 2020; 35(6): 1302-1305.

2. ALHOGBANI T. Acute myocarditis associated with novel middle east respiratory syndrome coronavirus. Ann Saudi Med. 2016; 36: 78-80.

3. BRASIL. Ministério da Saúde. Portaria MS/GM nํ 188, de 3 de fevereiro de 2020. Declara Emergência em Saúde Pública de importância Nacional (ESPIN) em decorrência da Infecção Humana pelo novo Coronavírus (2019-nCoV). Diário Oficial da União, Brasília (DF), 2020. Disponível em: https://www.in.gov.br/en/web/dou/-/portaria-n-188-de-3de-fevereiro-de-2020-241408388.

4. BRIZOLA J, et al. Revisão da literatura e revisão sistemática da literatura. Revista de Educação do Vale do ArinosRELVA, 2016 3(2).

5. CERAOLO C, GIORGI FM. Genomic variance of the 2019-nCoV coronavirus. J Med Virol. 2020; 92: $522-528$.

6. CHAVEZ S, et al. Coronavirus disease (COVID-19): a primer for emergency physicians. American Journal of Emergency Medicine. 2020.

7. CHEN C, et al. SARS-CoV-2: a potential novel etiology of fulminant myocarditis. Herz. 2020; 45(3): $230-232$.

8. CHEN J, et al. Findings of acute pulmonary embolism in COVID-19 patients. Lancet Infectious Disease. Clinical and Applied Thrombosis/Hemostasis; 2020 26:1076029620936772.

9. CHEN T, et al. Clinical characteristics of 113 deceased patients with coronavirus disease 2019: retrospective study. BMJ. 2020; 368. 
10. CHEN Y, et al. Structure analysis of the receptor binding of 2019-nCoV. BiochemBiophys Res Commun. 2020; 17; 525(1): 135-40.

11. CHENG P, et al. Cardiovascular Risks in Patients with COVID-19: Potential Mechanisms and Areas of Uncertainty. Relatóriosatuais de cardiologia, 2020; 22(5).

12. COUTARD B, et al. The spike glycoprotein of the new coronavirus 2019- nCoV contains a furin-like cleavage site absent in CoV of the same clade. Antiviral Res. 2020; 176: 104742.

13. DANZI GB, et al. Acute pulmonary embolism and COVID-19 pneumonia: a random association? Eur Heart J. 2020.

14. DRIGGIN E, et al. Cardiovascular considerations for patients, health care workers, and health systems during the coronavirus disease 2019 (COVID-19) pandemic. J Am Coll Cardiol; 2020.

15. ELFIKY AA. Anti-HCV, nucleotide inhibitors, repurposing against COVID-19. Life Sci. 2020; 248

16. FACONTI L, et al. Cardiovascular disease, heart failure and COVID-19. Journal of the Renin-AngiotensinAldosterone System, 2020; 20(2).

17. FERRARI F. COVID-19: dados atualizados e sua relação com o sistema cardiovascular. Arquivos Brasileiros de Cardiologia, 2020; 114.

18. GUO T, et al. Cardiovascular implications of fatal outcomes of patients with coronavirus disease 2019 (COVID-19). JAMA Cardiol. 2020.1017.

19. GUZIK TJ, et al. COVID-19 and the cardiovascular system: implications for risk assessment, diagnosis, and treatment options. Cardiovasc Res. 2020; 116(10): 1666-1687.

20. HANFF TC, et al. Is there an association between COVID-19 mortality and the renin-angiotensin system: a call for epidemiologic investigations. Clin Infect Dis. 2020.

21. HUANG C, et al. "Clinical features of patients infected with 2019 novel coronavirus in Wuhan, China." Lancet (London, England). 2020; 395(10223).

22. LIU K, et al. Clinical characteristics of novel coronavirus cases in tertiary hospitals in Hubei Province. Chin Med J (Engl). 2020.

23. LIU K, et al. Clinical and biochemical indexes from 2019-nCoV infected patients linked to viral loads and lung injury. Sci China Life Sci. 2020; 63: 364-374.

24. LONG B, et al. Cardiovascular complications in COVID-19. Am J Emerg Med. 2020; 38(7)

25. MARTINS JDN, et al. As implicações da COVID-19 no sistema cardiovascular: prognóstico e intercorrências. J Health BiolSci. $2020 \mathrm{~J} ; 8(1): 1-9$

26. ORGANIZAÇÃO MUNDIAL DE SAÚDE (OMS). Coronavirus disease (COVID-19) outbreak. Geneva: World Health Organization; 2021. Disponível em: https://www.paho.org/pt/brasil; acessado em: 20/02/2021.

27. PAGE RL, et al. Drugs that may cause or exacerbate heart failure: a scientific statement from the American Heart Association. Circulation. 2016; 134: e32-e69.

28. RUAN Q, et al. Clinical predictors of mortality due to COVID-19 based on an analysis of data of 150 patients from Wuhan, China. Intensive Care Med. 2020: 1-3.

29. SAVALAN BF, et al. Myocardial injury and COVID-19: Possible mechanisms. Life Sciences. 2020, 253(117723).

30. SHI S, et al. Association of cardiac injury with mortality in hospitalized patients with COVID-19 in Wuhan, China. JAMA Cardiology; 2020; 5(7): 802-810.

31. STILLWELL SB, et al. Searching for theEvidence. Am J Nurs [Internet]. 2010; 110(5): 41-7.

32. TANG N, et al. Abnormal coagulation parameters are associated with poor prognosis in patients with novel coronavirus pneumonia. J ThrombHaemost. 2020; 18(4): 844-847.

33. TIKELLIS C, et al. Angiotensin-converting enzyme 2 is a key modulator of the renin-angiotensin system in cardiovascular and renal disease. CurrOpin Nephrol Hypertens. 2011; 20(1): 62-8.

34. TONNESMANN E, et al. Chloroquine cardiomyopathy - a review of the literature. Immuno pharma collmmunotoxicol. 2013; 35: 434-442.

35. WANG D, et al. Clinical Characteristics of 138 Hospitalized Patients With 2019 Novel Coronavirus-Infected Pneumonia in Wuhan, China. JAMA. 2020; 323(11): 1061-1069.

36. WELT FGP, et al. from the American College of Cardiology's (ACC) Interventional Council and the Society of Cardiovascular Angiography and Intervention (SCAI) Catheterization laboratory considerations during the coronavirus (COVID-19) pandemic: from ACC's Interventional Council and SCAI. 2020.

37. XIE Y, et al. COVID-19 complicated by acute pulmonary embolism. Radiology: Cardiothoracic Imaging. $2020 ; 2$

38. ZHENG YY, et al., COVID-19 and the cardiovascular system. Nat Rev Cardiol. 2020 (17): 259-260.

39. ZHOU F, et al. Clinical course and risk factors for mortality of adult inpatients with COVID-19 in Wuhan, China: a retrospective cohort study. Lancet. 2020; 395(10229): 1054-1062.

40. ZOU X, et al. Single-cell RNA-seq data analysis on the receptor ACE2 expression reveals the potential risk of different human organs vulnerable to 2019-nCoV infection. Front Med. 2020 14(2): 185-92. 